\title{
Concentric Anchor-Beacons (CAB) Localization for Wireless Sensor Networks
}

\author{
Vijayanth Vivekanandan and Vincent W.S. Wong \\ Department of Electrical and Computer Engineering, \\ The University of British Columbia, Vancouver, Canada \\ e-mail: \{vijayv, vincentw\}@ece.ubc.ca
}

\begin{abstract}
Many applications in wireless sensor networks require sensor nodes to obtain their absolute or relative geographical positions. Although various localization algorithms have been proposed recently, most of them require nodes be equipped with range-determining hardware to obtain distance information. In this paper, we propose a concentric anchor-beacons (CAB) localization algorithm for wireless sensor networks. CAB is a range-free approach and uses a small number of anchor nodes. Each anchor emits beacons at different power levels. From the information received by each beacon heard, nodes determine which annular ring they are located within each anchor. Each node uses the approximated center of intersection of the rings as its position estimate. Simulation results show that the estimation error reduces by half when anchors transmit beacons at two different power levels instead of at a single level. CAB also gives a lower estimation error than other range-free localization schemes (e.g., Centroid, APIT) when the anchor-to-node range ratio is less than four.
\end{abstract}

\section{INTRODUCTION}

Significant advances in hardware technology have led to the miniaturization of devices capable of communication with each other. Wireless sensor networks consist of hundreds or thousands of tiny nodes that are deployed to monitor and gather data in a target geographical area. These nodes have limited processing capabilities and energy in which to operate. Wireless sensor networks are envisioned to allow for ease of deployment through redundancy and ad hoc placement. Applications such as remote surveillance or habitat monitoring require sensor nodes to obtain their absolute or relative geographical positions. When an event occurs (or a stimulus being detected), the sensor nodes can forward the data information along their coordinates.

Various centralized [1][2] and distributed [3][4] localization algorithms have been proposed recently. With respect to robustness and energy efficiency, distributed algorithms are preferred over centralized schemes. The localization algorithms can further be divided into range-based [4][5], anglebased [6][7], and range-free [8][9] approaches. Range-based schemes assume that sensor nodes have the ability to obtain distance estimates to other nodes. In angle-based schemes, the relative angular information between nodes is required. Range-free approaches assume that no specialized angle or range-determining hardware is necessary for the sensor nodes. To determine the absolute geographical location, most of the localization algorithms also assume the use of special anchor nodes. Each anchor may equip with a GPS (Global Positioning System) receiver to obtain its absolute position information.

Although both range-based and angle-based approaches provide a lower estimation error than the range-free approaches, they require special hardware for sensor nodes to obtain relatively accurate distance (or angle) measurements to other nodes and anchors. This may not be cost-effective for applications which require hundreds of sensor nodes over a large coverage area. Our work focuses on improving distributed range-free algorithms with higher accuracy.

In this paper, we propose a concentric anchor-beacons (CAB) localization algorithm for wireless sensor networks [10]. CAB is a distributed range-free approach and uses a small percentage of anchor nodes. Each anchor emits beacon signals at different power levels. Each beacon carries information including the anchor's position, its power level, and the estimated maximum distance the beacon can travel. Nodes listen and record which anchors they can hear the beacons from and at which power levels. From the information received by each beacon heard, nodes determine which annular ring they are located within each anchor. Each node uses the approximated center of intersection of the rings as its position estimate.

$\mathrm{CAB}$ requires no specialized range-determining hardware in the sensor nodes, and relies only on node-anchor communication to localize the nodes. This simplifies the distributed operation and is more energy-efficient since neighboring sensor nodes do not need to exchange information.

Simulation experiments are conducted to evaluate the performance of $\mathrm{CAB}$ localization algorithm by varying the number of anchors heard, anchor-to-node range ratio, and radio pattern degree of irregularity. Simulation results show that the estimation error reduces by half when anchors transmit beacons at two different power levels periodically instead of a single power level. In addition, we also compare $\mathrm{CAB}$ with two other range-free localization algorithms: Centroid [8] and APIT (Approximated Point-In-Triangulation) [9]. Results show that $\mathrm{CAB}$ provides a higher accuracy than Centroid. $\mathrm{CAB}$ gives a lower estimation error than APIT when the anchor-to-node range ratio is less than four.

This paper is organized as follows. The related work is summarized in Section II. The CAB localization algorithm is described in Section III. The performance evaluation of CAB as well as the comparisons with APIT and Centroid are presented in Section IV. Conclusions are given in Section V. 


\section{RELATED WORK}

In this section, we provide an overview of several localization algorithms. Survey papers can be found in [11][12].

In the centralized convex optimization scheme [1], the intersection of all neighboring nodes' communication range is considered. A bounding box is constructed and the centroid is taken as the position estimate of the node. Algorithms based on Multidimensional Scaling (MDS) [2][5] can operate in both range-free and range-based scenarios. They are very accurate and only 3 or 4 anchor nodes are required. However, they also require considerable amount of overhead for communication and computation.

Schemes based on anchor location propagation throughout the network include [3][4][13]. The anchor's position as well as hop counts or distance from the node are used to bound or laterate the location of the node. At least three anchors are needed for nodes to obtain a unique position estimate. The estimation accuracy depends on the accuracy of the distance information used in lateration or bounding.

For the angle-based schemes, the direction between nodes can be determined by using antenna-arrays. In [6], nodes which have at least 3 bearings to anchors can triangulate their positions. Both range and angle information is used in [7] in order to necessitate only one anchor's information for position estimation.

Several schemes have explored the use of mobile anchors and nodes. In [14], a single mobile anchor traverses the network and allows stationary sensor nodes to compute their location estimates based on at least three neighboring nodes' locations. Multiple mobile anchors are used in [15]. In [16], both anchors and sensor nodes are mobile. The Monte Carlo algorithm is used for localization. In [17], an extended Kalman filter (EKF)-based state estimator is used in tandem with mobile robots for localization.

In the range-free Centroid algorithm [8], anchors are placed in a grid configuration. Each sensor identifies which anchors it can hear from, and then estimates its location as the average of the coordinates of all anchors heard. In the APIT (Approximated Point-In-Triangulation) algorithm [9], each node first determines if it is within a particular triangle formed by a set of anchors within anchor range. The position is estimated to be the center of intersection of all triangles in which the node has identified to be within.

The work in [18] considered the possibility of quantized received signal strength (RSS) and presented mathematical analysis of the accuracy with varying levels of quantization. The performance comparisons between ROCRSSI and APIT schemes are given in [19].

\section{CONCENTRIC ANCHOR-BEACONS (CAB) LOCALIZATION AlgORITHM}

In this section, we begin with a discussion of the motivations and assumptions. It is followed by the description of the $\mathrm{CAB}$ localization algorithm. We then discuss the advantages and limitations of our proposed scheme.

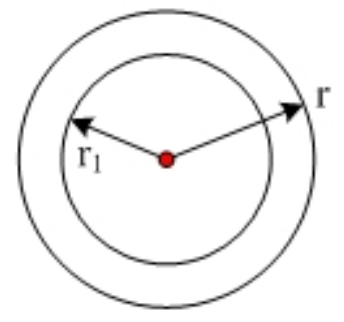

(a)

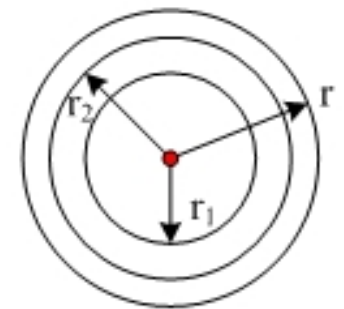

(b)
Fig. 1. Anchor beacon transmission ranges with (a) beacon signals with two power levels; and (b) beacon signals with three power levels.

\section{A. Motivations and Assumptions}

Although distributed range-based algorithms have a higher accuracy than the distributed range-free approaches in general, the range-free approaches are more cost effective. In our work, we focus on the design of a distributed range-free localization algorithm that has a high accuracy and does not require communication between neighboring sensor nodes.

In our proposed scheme, each sensor node estimates its position solely based on the information gathered directly from the anchor nodes. Since our scheme does not depend on neighboring sensor node communication, it is independent of network connectivity. Sensor nodes do not require any special range-determining hardware for localization. On the other hand, anchors are equipped with GPS modules. Thus, anchor nodes are more costly, consume more energy, and are larger in size than normal sensor nodes. In addition, as in the case of some other schemes (e.g., [9]), anchors are assumed to have larger communication range than normal sensor nodes. The anchor-to-node range (ANR) ratio is equal to the maximum communication range of an anchor divided by the communication range of a sensor node.

The main difference between $\mathrm{CAB}$ and other range-fee localization approaches is that anchors transmit beacon signals at different power levels. This requirement is feasible in the current wireless sensor networks. For example, the Mica2 mote sensor nodes have a range of 18 meters for transmission power of $-10 \mathrm{dbm}$, and 50 meters for $0 \mathrm{dbm}$ [20].

Ideally, the different power levels divide the possible transmission ranges of an anchor into circle and rings. As shown in Figure 1, the lowest power level creates a circular coverage area, and the following higher levels are distinguished by rings emanating from this lowest level.

In our work, we assume that the area of the inner-most circle and the rings are all the same. That is, in Figure 1(a), the circle with radius $r_{1}$ has the same area as of the ring outside that circle. The relationship between the beacon transmission ranges and the maximum transmission range is given by:

$$
r_{i}=\sqrt{\frac{i}{m}} r \quad i=1,2, \cdots, m
$$

where $m$ denotes the total number of different beacon power levels; $i$ denotes the beacon number starting from the lowest 
power level (or transmission range); $r$ denotes the maximum range that an anchor can transmit at the corresponding maximum power level $P_{\max }$.

In free-space propagation environment, the required power to transmit at a distance $d$ is directly proportional to the square of the distance. In general, the transmit power is directly proportional to the $n^{\text {th }}$ power of the distance, where $n$ denotes the path loss exponent. We have:

$$
P_{i}=\left(\frac{i}{m}\right)^{\frac{n}{2}} P_{\max } \quad i=1,2, \cdots, m
$$

where $P_{i}$ represents the transmit power for beacon $i$ in terms of the maximum transmit power $P_{\max }$.

Consider an example in Figure 1(a). If the sensor node can hear the beacons with power levels $P_{1}$ and $P_{2}$, then the distance between the anchor and the node is less than $r_{1}$. That is, the sensor node lies within the inner-most circle, On the other hand, if the node can only hear the beacons with power level $P_{2}$, then it lies within the ring.

Before deployment, measurement is necessary to relate the transmission power $P_{i}$ and coverage range $r_{i}$. This is important in order to ensure the accuracy of the range-free approach.

\section{B. CAB Algorithm}

We now describe the $\mathrm{CAB}$ localization algorithm in details. Each anchor transmits the beacon signals at varying power levels consecutively. The time between two beacon transmissions follows a general distribution with mean equals to $T$. This reduces the likelihood of collision of beacon signals transmitted by two different neighboring anchors simultaneously. Each beacon signal packet includes the anchor's ID, anchor's location, and the transmit power level $P_{i}$ information. Each node listens for beacons and collects the anchor's information. For each beacon heard, the sensor node determines which region of the anchor's concentric transmission circles it lies within. Figure 2 shows an example with a sensor node surrounded by three anchors. Each anchor transmit beacons periodically at two different power levels. The corresponding information table collected by the sensor is shown in Table I.

Depending on the percentage of anchors deployed, each sensor node can hear multiple beacons from different anchors. For computational simplicity, information from at most three neighboring anchors is used to estimate a sensor's location. In order to minimize the area of intersection of the region of each anchor that the node lies within and to increase the accuracy of the position's estimate, the three anchors that are farthest apart are being chosen. This is achieved by calculating all the possible triangular areas that made up of three anchors heard, and by choosing the three anchors that form the largest triangle.

For the beacons sent by each anchor, the sensor node is assumed to be within a particular region. This region is bound by either two equations of circles (for the ring case) or just one equation of a circle (for the inner-most region of the anchor). The last column in Table 1 shows an example of the regions that the sensor node lies within.

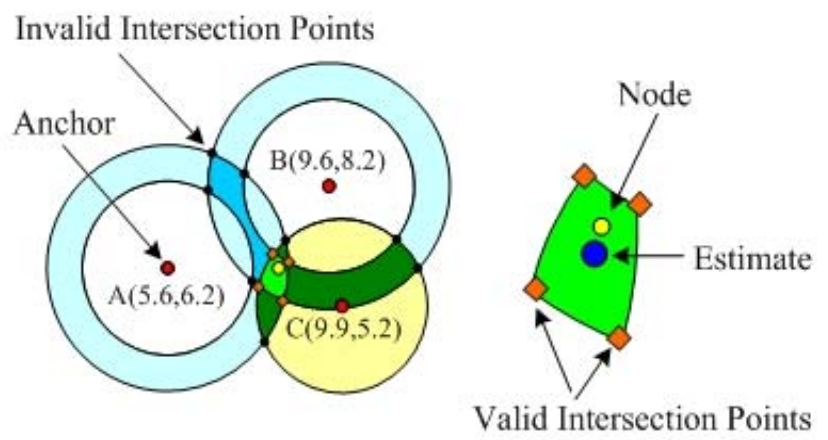

Fig. 2. An example of localization using CAB.

TABLE I

INFORMATION COLLECTED BY A SENSOR FROM ITS ANCHORS

\begin{tabular}{|c|c|c||c|}
\hline $\begin{array}{c}\text { Anchor } \\
j\end{array}$ & $\begin{array}{c}\text { Position } \\
\left(x_{j}, y_{j}\right)\end{array}$ & $\begin{array}{c}\text { Beacons Transmit } \\
\text { Power Info }\end{array}$ & $\begin{array}{c}\text { Constraining } \\
\text { Region }\end{array}$ \\
\hline$A$ & $(5.6,6.2)$ & $P_{2}$ & $\left(r_{1}-r\right)$ ring \\
\hline$B$ & $(9.6,8.2)$ & $P_{2}$ & $\left(r_{1}-r\right)$ ring \\
\hline$C$ & $(9.9,5.2)$ & $P_{1}, P_{2}$ & $r_{1}$ circle \\
\hline
\end{tabular}

Given the three chosen anchors, two of them are selected at a time to calculate the intersection points. The valid intersection points satisfy all three anchors' constraint regions. The invalid intersection points are those that do not lie within the other anchor's constraint region. Consider the example in Figure 2. Let $\left(x_{A}, y_{A}\right),\left(x_{B}, y_{B}\right),\left(x_{C}, y_{C}\right)$ denote the positions of anchors $A, B$, and $C$, respectively. Let $I$ denote the set of intersection points. For each point $(x, y) \in I$, it is a valid intersection point if the following constraints are satisfied:

$$
\begin{array}{r}
r_{1} \leq \sqrt{\left(x_{A}-x\right)^{2}+\left(y_{A}-y\right)^{2}} \leq r \\
r_{1} \leq \sqrt{\left(x_{B}-x\right)^{2}+\left(y_{B}-y\right)^{2}} \leq r \\
\sqrt{\left(x_{C}-x\right)^{2}+\left(y_{C}-y\right)^{2}} \leq r_{1}
\end{array}
$$

The final position estimate is taken as the average of all the valid intersection points. Figure 2 shows the estimated position determined by four valid intersection points.

The above description of the CAB localization algorithm is also valid when the sensor node only receives beacon signals from two neighboring anchors. The valid intersection points can be determined from the intersection of those rings (or circles). The final position estimate is taken as the average of the valid intersection points. On the other hand, if the sensor node receives beacon signals from only one anchor, then a random coordinate within the ring that the sensor node resides will be chosen as the position estimate.

\section{Discussion}

There are three distinct advantages of $\mathrm{CAB}$ localization algorithm. First of all, $\mathrm{CAB}$ is distributed and is simple to implement. For the anchors, their only task is to transmit beacons signals with different power levels. For each sensor 
node, the determination of the intersection points from three chosen anchors as well as the position estimate by averaging are not computationally intensive. Second, no information exchange between neighboring sensors is necessary. This reduces the energy requirement for localization. In addition, $\mathrm{CAB}$ has a higher accuracy than some other range-free localization algorithms. Simulation comparisons will be presented in the next section.

For the qualitative comparisons with some other localization algorithms, APIT [9] requires communication between neighboring nodes for the exchange of tabular information of nearby anchors. $\mathrm{CAB}$ does not require that procedure and achieves better results under smaller anchor-to-node range ratio. In comparison to Centroid [8] which requires a gridbased deployment, $\mathrm{CAB}$ is able to perform sufficiently well in ad hoc deployments. Whereas ring sizes are determined from RSS values in ROCRSSI [19], the rings in CAB are preset according to the number of power levels to be used by the anchors. No communication is required between anchors in CAB.

Our scheme is not without limitations. Being solely dependent on anchor nodes for position estimation, the accuracy depends on the percentage of nodes deployed that are anchors. This percentage can be decreased by increasing the maximum radio range of the anchors. However, this results in less accuracy since the intersection areas become larger. Also, since our scheme's computation relies on a circular radio model, it can be affected by irregular radio propagation, to which other range-free schemes are relatively immune. In the next section, we will also present the results of our scheme under different degrees of radio patterns.

\section{Performance EVAluation AND COMPARISON}

In this section, we present the performance evaluation of $\mathrm{CAB}$ as well as the comparisons with APIT [9] and Centroid [8] algorithms. All three algorithms are simulated in Matlab 7.0 on a $3.06 \mathrm{GHz}$ Pentium IV processor. The wireless sensor network consists of 280 nodes and varying number of anchors is randomly placed. The network topology is a square of side $10 R$ by $10 R$, where $R$ is the sensor node communication range. The average connectivity among nodes is set to eight.

We use the technique in [9] to model the irregular radio pattern. In this model, all nodes within half the maximum transmit radio range of anchors are guaranteed to hear from the anchor, whereas nodes between the maximum radio range and half of that range may or may not hear from the anchor depending on the radio pattern in that direction. The degree of irregularity (DOI) parameter is defined as the maximum radio range variation per unit degree change in direction. Examples of different DOI values of this irregular radio pattern model are shown in Figure 3.

For CAB, we assume a path loss exponent $(n)$ of two. Unless stated otherwise, beacons are being transmitted under two different power levels (i.e., $m=2$ ) periodically. The anchor-to-node range (ANR) ratio is set at three. The DOI

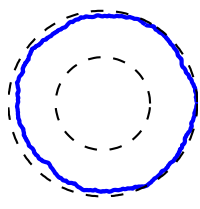

$\mathrm{DOI}=0.01$

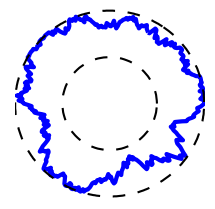

$\mathrm{DOI}=0.05$

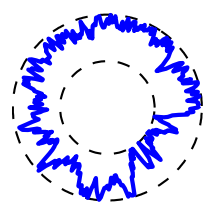

$\mathrm{DOI}=0.10$
Fig. 3. Irregular radio patterns for different values of DOI.

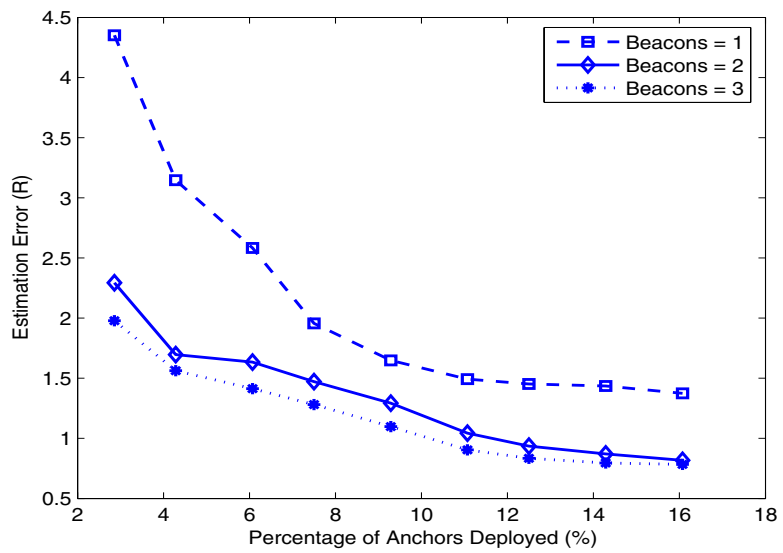

Fig. 4. Average estimation error under different number of power levels of the beacons. (ANR $=3$, DOI $=0.05$ )

value is set at 0.05 . The estimation errors are normalized with respect to the sensor node range $(R)$.

\section{A. Performance of $C A B$}

Figure 4 shows the accuracy gain of $\mathrm{CAB}$ by increasing the number of power levels of the beacons (i.e., increase of $m$ ). When beacons are being transmitted at a single power level $(m=1)$, the intersection area is constructed by determining the intersections of three circles centered at their corresponding anchors. It is clear that with two different power levels $(m=2)$, it reduces the intersection area to intersections of rings and circles. Figure 4 shows that the estimation error reduces by at least $0.44 R$ when $m$ increases from 1 to 2, a significant improvement. Notice that when the number of different power levels increases to 3 (or higher), the performance improvement is marginal. This is due to the fact that when $m$ is further increased, the anchor coverage area is subdivided into a circle and more concentric rings. The irregular radio pattern model introduces more errors to the rings with smaller ring width.

Figure 5 shows the performance comparison under two different $r_{1}$ radii. Although we first tried using anchor beacons with power levels corresponding to equal ring widths (i.e., $r_{1}=0.5 r$ ), we quickly identified that the equal area approach (i.e., $r_{1}=0.707 r$ ) achieved more accurate results. The estimation error is reduced by $0.32 R$ on average. However, for larger $m$ and DOI values, an increase of the width of the outer rings may be preferred to guarantee accurate identification of which region the node lies within. 


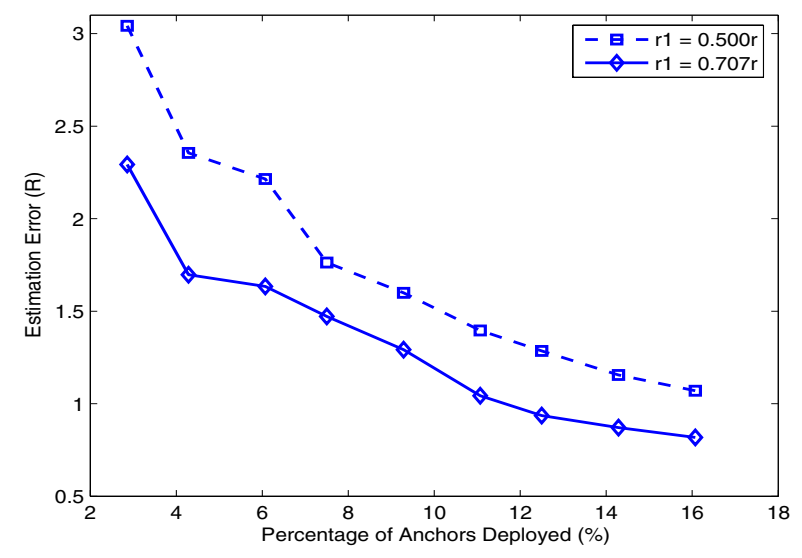

Fig. 5. Performance gain of equal area $\mathrm{CAB}$ versus equal radius $\mathrm{CAB}$. $(m=2, \mathrm{ANR}=3, \mathrm{DOI}=0.05)$

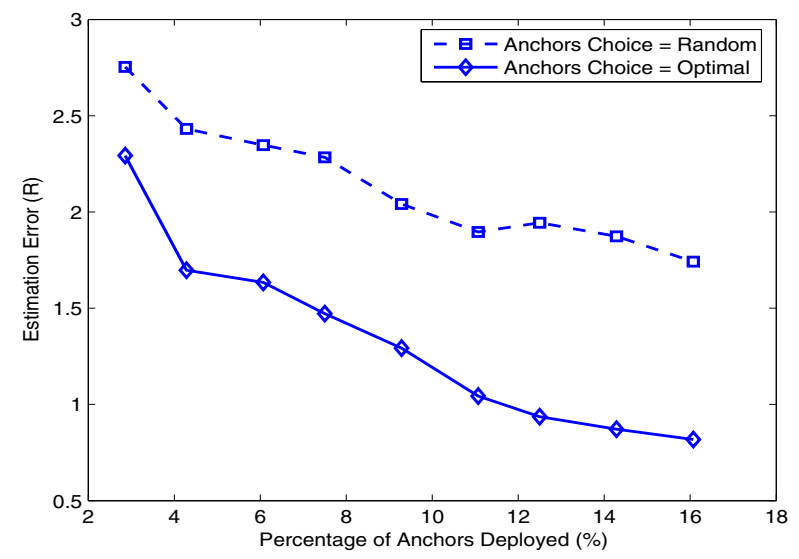

Fig. 6. Comparison of estimation error using randomly heard anchors versus optimally chosen anchors. $\left(m=2, r_{1}=0.707 r, \mathrm{ANR}=3, \mathrm{DOI}=0.05\right)$

It is possible that a sensor node may receive beacon signals from more than three anchors. In $\mathrm{CAB}$, only three neighboring anchors are used for localization. Figure 6 shows the comparison between two different ways of choosing those three neighboring anchors. For the case of random, the three anchors heard with the lowest IDs are chosen. For the case of optimal, the three anchors which form the largest triangle are chosen. Results in Figure 6 show that the optimal approach provides a much lower estimation error than the random choice. As an example, when the percentage of anchors deployed is $11 \%$, the optimal choice provides an estimation error $0.95 R$ lower than the random choice on average. In addition, for the optimal choice, the estimation error decreases when the percentage of anchors deployed increases. This is expected since there are more anchors from which to choose.

The choice of using only three anchors for position estimation is to reduce the computational complexity, since considering more anchors results in many more intersection points to be computed. We are aware that choosing the anchors that result in the largest triangle region does not always guarantee the smallest coverage intersection, since the

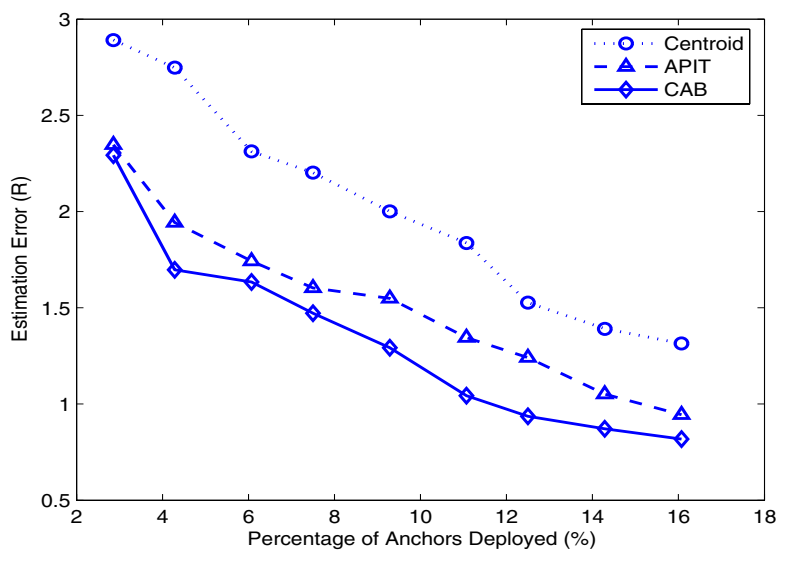

Fig. 7. Comparison between Centroid, APIT and CAB by increasing the percentage of anchors deployed. $\left(m=2, r_{1}=0.707 r\right.$, ANR $=3$, DOI $=$ $0.05)$

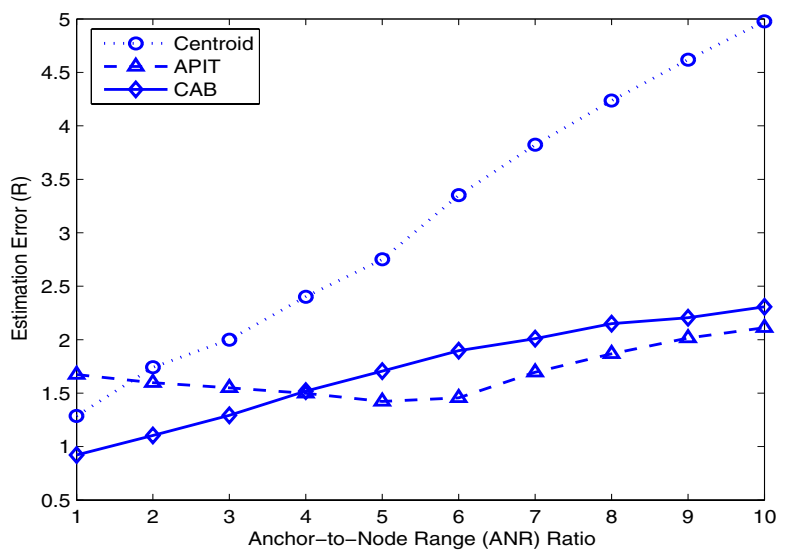

Fig. 8. Comparison between Centroid, APIT and CAB for varying levels of ANR. $\left(m=2, r_{1}=0.707 r\right.$, DOI $\left.=0.05\right)$

intersection also depends on the size of the circle or ring constraining the position of the node. However, further results show that choosing the three anchors that form the largest triangle is sufficient in achieving good accuracy [10].

\section{B. Comparisons between CAB, APIT, and Centroid}

Figure 7 shows the position estimation errors as a function the percentage of anchors deployed. $\mathrm{CAB}$ has a better performance than both APIT and Centroid. As an example, when the percentage of anchors deployed is $16 \%, \mathrm{CAB}, \mathrm{APIT}$, and Centroid achieve $0.81 R, 0.94 R$, and $1.31 R$ accuracy, respectively. Note that the performance of $\mathrm{CAB}$ can further be improved by utilizing information from more than three anchors at the expense of a higher computation complexity.

Figure 8 shows the results of the estimation error as a function of ANR ratio. The percentage of anchors deployed is $9 \%$. As the ANR value increases, this results in loss of accuracy in all schemes. In the Centroid scheme, nodes can now hear anchors that are farther, and result in a more coarse grained estimation of position. In the APIT scheme, the ANR 


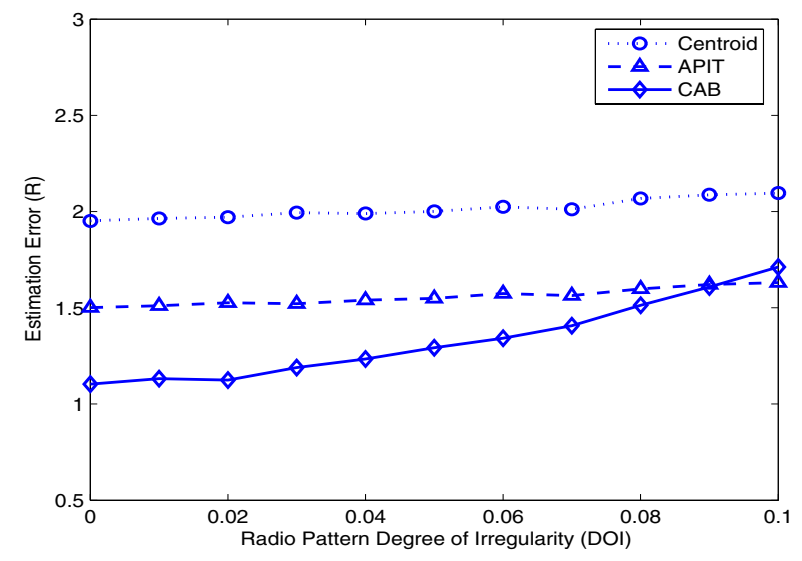

Fig. 9. Comparison between Centroid, APIT and CAB under different DOI values. $\left(m=2, r_{1}=0.707 r, \mathrm{ANR}=3\right)$

actually improves the accuracy until ANR equals to 5. The error then increases with higher ANR values. This unique behavior can be attributed to the InToOut error identified by in [9] which is more significant at low ANR values and diminishes with increasing ANR. The CAB algorithm only relies on anchor information and thus increases in error as ANR increases. The higher ANR values result in larger ring areas which in turn create larger intersections within which the node estimate is taken.

Figure 8 also shows that APIT outperforms CAB for ANR greater than four, though the difference of performance gain is marginal as the ANR increases further. Note that in APIT, each sensor node consumes additional energy for the exchange of information between neighboring nodes. In $\mathrm{CAB}$, information exchange between neighboring nodes is not necessary. In other words, the accuracy of $\mathrm{CAB}$ does not depend on the average node degree or the connectivity information.

Figure 9 shows the effects of irregular radio propagation on the accuracy of the range-free schemes. The percentage of anchors deployed is $9 \%$. Due to the use of the fixed empirical range values for different transmit power levels of the beacons, $\mathrm{CAB}$ is more sensitive to the irregular radio pattern than Centroid and APIT. When DOI values less than 0.09, CAB outperforms the other two schemes. However, for higher DOI values, APIT has a better performance than CAB.

Based on the above results, we suggest that the following parameters be used for the implementation of CAB localization algorithm: $m=2, r_{1}=0.707 r, A N R \leq 3$, percentage of anchors deployed between $9 \%$ and $16 \%$.

\section{CONCLUSIONS}

In this paper, we proposed the $\mathrm{CAB}$ localization algorithm for wireless sensor networks. $\mathrm{CAB}$ is a distributed range-free approach and do not require information exchange between neighboring sensors. It has a low computation overhead and is simple to implement. CAB uses anchors that broadcast beacon signals at varying power levels periodically. This allows each sensor node to identify which annular ring, centered at the anchor, the node resides in. The estimated position of the node is taken as the average of all the valid intersection points. Simulation results show that $\mathrm{CAB}$ provides a lower position estimation error than APIT and Centroid under a wide range of conditions. Further work includes the implementation of $\mathrm{CAB}$ via testbed prototyping.

\section{ACKNOWLEDGMENT}

This work is supported by the Natural Sciences and Engineering Research Council of Canada under grant number 261604-03.

\section{REFERENCES}

[1] L. Doherty, K. Pister, and L. Ghaoui, "Convex position estimation in wireless sensor networks," in Proc. of IEEE Infocom, Anchorage, AK, April 2001.

[2] Y. Shang, W. Ruml, Y. Zhang, and M. Fromherz, "Localization from mere connectivity," in Proc. of ACM MobiHoc, Annapolis, MD, June 2003.

[3] K. Langendoen and N. Reijers, "Distributed localization in wireless sensor networks: A quantitative comparison," Computer Networks, vol. 43, Nov. 2003.

[4] D. Niculescu and B. Nath, "Ad-hoc positioning system," in Proc. of IEEE Globecom, San Antonio, TX, Nov. 2001.

[5] Y. Shang, W. Ruml, and Y. Zhang, "Improved MDS-based localization," in Proc. IEEE Infocom, Hong Kong, China, March 2004.

[6] D. Niculescu and B. Nath, "Ad-hoc positioning system (APS) using AOA," in Proc. of IEEE Infocom, San Francisco, CA, April 2003.

[7] K. Chintalapudi, A. Dhariwal, R. Govindan, and G. Sukhatme, "Ad-hoc localization using ranging and sectoring," in Proc. of IEEE Infocom, Hong Kong, China, March 2004.

[8] N. Bulusu, J. Heidemann, and D. Estrin, "GPS-less low cost outdoor localization for very small devices," IEEE Personal Communications Magazine, vol. 7, no. 5, pp. 28-34, Oct. 2000.

[9] T. He, C. Huang, B. Lum, J. Stankovic, and T. Adelzaher, "Range-free localization schemes for large scale sensor networks," in Proc. of ACM MobiCom, San Diego, CA, Sept. 2003.

[10] V. Vivekanandan, "Localization algorithms for wireless sensor networks," M.A.Sc. Thesis, The University of British Columbia, Vancouver, BC, Canada, Dec. 2005.

[11] D. Niculescu, "Positioning in ad hoc sensor networks," IEEE Network Magazine, vol. 18, no. 4, pp. 24-29, July 2004.

[12] A. Savvides, M. Srivastava, L. Girod, and D. Estrin, "Localization in sensor networks," book chapter in Wireless Sensor Networks, Springer, 2005.

[13] C. Savarese, J. Rabaey, and K. Langendoen, "Robust positioning algorithms for distributed ad-hoc wireless sensor networks,", in Proc. of USENIX Technical Annual Conference, Monterey, CA, June 2002.

[14] M. Sichitiu and V. Ramadurai, "Localization of wireless sensor networks with a mobile beacon," in Proc. of IEEE MASS, Fort Lauderdale, FL, Oct. 2004.

[15] K.-F. Ssu, C.-H. Ou, and H. Jiau, "Localization with mobile anchor points in wireless sensor networks," IEEE Trans. on Vehicular Technology, vol. 54, pp. 1186-1197, May 2005.

[16] L. Hu and D. Evans, "Localization for mobile sensor networks," in Proc. of ACM MobiCom, Philadelphia, PA,, Sept. 2004.

[17] P. Pathirana, N. Bulusu, A. Savkin, and S. Jha, "Node localization using mobile robots in delay-tolerant sensor networks," IEEE Trans. on Mobile Computing, vol. 4, pp. 285-296, July/Aug. 2005.

[18] N. Patwari and A. Hero, "Using proximity and quantized RSS for sensor localization in wireless networks,", in Proc. of ACM WSNA, Sept. 2003.

[19] C. Liu and K. Wu, "Performance evaluation of range-free localization methods for wireless sensor networks," in Proc. of IEEE IPCCC, Phoenix, AZ, April 2005.

[20] G. Xing, C. Lu, Y. Zhang, Q. Huang, and R. Pless, "Minimum power configuration in wireless sensor networks," in Proc. of ACM MobiHoc, Urbana-Champaign, IL, May 2005. 